Commun. Korean Math. Soc. 28 (2013), No. 3, pp. 527-534

http://dx.doi.org/10.4134/CKMS.2013.28.3.527

\title{
ON A HYPERGEOMETRIC SUMMATION THEOREM DUE TO QURESHI ET AL.
}

\author{
Junesang Choi and Aruun K. Rathie
}

\begin{abstract}
We first aim at proving an interesting easily derivable summation formula. Then it is easily seen that this formula immediately yields a hypergeometric summation theorem recently added to the literature by Qureshi et al. Moreover we apply the main formulas to present some interesting summation formulas, whose special cases are also seen to yield the earlier known results.
\end{abstract}

\section{Introduction and preliminaries}

It is well known that the Chebyshev polynomials $T_{n}(x)$ and $U_{n}(x)$ of the first and second kind are the following special cases $\left(\alpha=\beta=-\frac{1}{2}\right.$ and $\alpha=\beta=\frac{1}{2}$, respectively) of the classical Jacobi polynomials $P_{n}^{(\alpha, \beta)}(x)$ defined by (see, for example, [3, pp. 169-170] and [5])

$$
P_{n}^{(\alpha, \beta)}(x):=\sum_{k=0}^{n}\left(\begin{array}{c}
n+\alpha \\
k
\end{array}\right)\left(\begin{array}{c}
n+\beta \\
n-k
\end{array}\right)\left(\frac{x-1}{2}\right)^{n-k}\left(\frac{x+1}{2}\right)^{k} .
$$

Also, in terms of the generalized hypergeometric series ${ }_{p} F_{q}$ defined by (see $[6$, p. 73]):

$$
\begin{aligned}
{ }_{p} F_{q}\left[\begin{array}{c}
\alpha_{1}, \ldots, \alpha_{p} ; z \\
\beta_{1}, \ldots, \beta_{q} ; z
\end{array}\right] & =\sum_{n=0}^{\infty} \frac{\left(\alpha_{1}\right)_{n} \cdots\left(\alpha_{p}\right)_{n}}{\left(\beta_{1}\right)_{n} \cdots\left(\beta_{q}\right)_{n}} \frac{z^{n}}{n !} \\
& ={ }_{p} F_{q}\left(\alpha_{1}, \ldots, \alpha_{p} ; \beta_{1}, \ldots, \beta_{q} ; z\right) \quad\left(p, q \in \mathbb{N}_{0}\right)
\end{aligned}
$$

where $(\lambda)_{\nu}$ denotes the Pochhammer symbol or the shifted factorial, since

$$
(1)_{n}=n ! \quad\left(n \in \mathbb{N}_{0}:=\mathbb{N} \cup\{0\} ; \mathbb{N}:=\{1,2,3, \ldots\}\right),
$$

Received August 7, 2012.

2010 Mathematics Subject Classification. Primary 33B10, 33C05; Secondary 33C65.

Key words and phrases. gamma function, Pochhammer symbol, hypergeometric function, generalized hypergeometric function, Chebyshev polynomials of the first and second kind, Jacobi polynomials. 
which is defined (for $\lambda, \nu \in \mathbb{C}$ ), in terms of the familiar gamma function $\Gamma$, by

$$
(\lambda)_{\nu}:=\frac{\Gamma(\lambda+\nu)}{\Gamma(\lambda)}=\left\{\begin{array}{cl}
1 & (\nu=0 ; \lambda \in \mathbb{C} \backslash\{0\}) \\
\lambda(\lambda+1) \cdots(\lambda+n-1) & (\nu=n \in \mathbb{N} ; \lambda \in \mathbb{C}),
\end{array}\right.
$$

it being understood conventionally that $(0)_{0}:=1$ and $\mathbb{C}$ the set of complex numbers, (1.1) can be written as

$$
P_{n}^{(\alpha, \beta)}(x)=\left(\begin{array}{c}
n+\alpha \\
n
\end{array}\right){ }_{2} F_{1}\left[\begin{array}{r}
-n, n+\alpha+\beta+1 ; \\
\left.\alpha+1 ; \frac{1-x}{2}\right] .
\end{array}\right.
$$

In fact, we have the following relationships (see, for example, [8, p. 125] and $[5])$ :

$$
T_{n}(x)=\left(\begin{array}{c}
n-\frac{1}{2} \\
n
\end{array}\right)^{-1} P_{n}^{\left(-\frac{1}{2},-\frac{1}{2}\right)}(x)
$$

and

$$
U_{n}(x)=\frac{1}{2}\left(\begin{array}{c}
n+\frac{1}{2} \\
n+1
\end{array}\right)^{-1} P_{n}^{\left(\frac{1}{2}, \frac{1}{2}\right)}(x) .
$$

Moreover, upon setting $x=\cos \theta$ in Equations (1.5) and (1.6), it is easily seen that (see, for example, [8, p. 71, Equation 1.8(10)] and [5])

$$
T_{n}(\cos \theta)=\cos (n \theta)
$$

and

$$
U_{n}(\cos \theta)=\frac{\sin [(n+1) \theta]}{\sin \theta} .
$$

Also, for the Chebyshev polynomials $T_{n}(x)$ and $U_{n}(x)$ defined by Equations (1.5) and (1.6), it is known that (see, for example, [4, p. 468, Entries 7.3.1(209) and $7.3 .1(211)]$ )

$$
{ }_{2} F_{1}\left[\begin{array}{c}
-\frac{n}{2},-\frac{n}{2}+\frac{1}{2} ; \\
\frac{1}{2} ;
\end{array}\right]=(1-x)^{\frac{n}{2}} T_{n}\left(\frac{1}{\sqrt{1-x}}\right)
$$

and

$$
{ }_{2} F_{1}\left[\begin{array}{r}
-\frac{n}{2},-\frac{n}{2}+\frac{1}{2} ; \\
\frac{3}{2} ;
\end{array}\right]=\frac{(1-x)^{\frac{n}{2}}}{n+1} U_{n}\left(\frac{1}{\sqrt{1-x}}\right)
$$

Upon setting $x=-\frac{b^{2}}{a^{2}}$ in Equations (1.9) and (1.10), recently Qureshi et al. [5, Equations (18) and (19)] obtained two interesting hypergeometric summation formulae given in the following theorem. 
Theorem 1. For $n \in \mathbb{N}_{0}$, we have

$$
\begin{aligned}
& \left.{ }_{2} F_{1}\left[\begin{array}{c}
-\frac{n}{2}, \frac{1-n}{2} ; \\
\frac{1}{2} ;
\end{array}\right] \frac{b^{2}}{a^{2}}\right]=\frac{\left(a^{2}+b^{2}\right)^{\frac{n}{2}}}{a^{n}} \cos (n \vartheta) \\
& \left(n \vartheta \neq \frac{2 \kappa+1}{2} \pi ; \kappa \in \mathbb{Z}\right)
\end{aligned}
$$

and

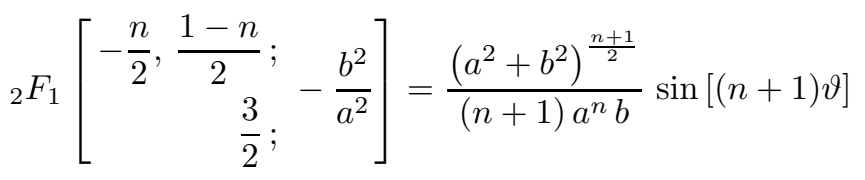

$$
\begin{aligned}
& ((n+1) \vartheta \neq \kappa \pi ; \kappa \in \mathbb{Z}),
\end{aligned}
$$

where $\mathbb{Z}$ denotes the set of integers and $\vartheta$ is given by

$$
\vartheta:= \begin{cases}\arctan \left(\frac{b}{a}\right) & \left(a, b \in \mathbb{R}_{+}\right) \\ \pi-\arctan \left(\frac{b}{|a|}\right) & \left(a \in \mathbb{R}_{-} ; b \in \mathbb{R}_{+}\right) \\ \arctan \left(\frac{|b|}{|a|}\right)-\pi & \left(a, b \in \mathbb{R}_{-}\right) \\ -\arctan \left(\frac{|b|}{a}\right) & \left(a \in \mathbb{R}_{+} ; b \in \mathbb{R}_{-}\right),\end{cases}
$$

$\mathbb{R}_{+}$and $\mathbb{R}_{-}$being the sets of positive and negative real numbers, respectively.

Here, in this paper, we aim at presenting an interesting easily derivable summation formula (2.1) involving the exponential and hypergeometric functions ${ }_{2} F_{1}$. Moreover, it is easily seen that our summation formula immediately yields Equations (1.11) and (1.12).

\section{Main result}

The main result to be established here is as in the following theorem.

Theorem 2. The following summation formula holds true.

$$
\begin{aligned}
& \sum_{n=0}^{\infty}\left(a^{2}+b^{2}\right)^{\frac{n}{2}} e^{i n \vartheta} \frac{x^{n}}{n !}=\sum_{n=0}^{\infty} \frac{a^{n} x^{n}}{n !}{ }_{2} F_{1}\left[\begin{array}{c}
-\frac{n}{2}, \frac{1-n}{2} ; \\
\frac{1}{2} ;
\end{array}\right] \\
& +i b \sum_{n=0}^{\infty} \frac{a^{n} x^{n+1}}{n !}{ }_{2} F_{1}\left[\begin{array}{r}
-\frac{n}{2}, \frac{1-n}{2} ; \\
\frac{3}{2} ;
\end{array}\right]
\end{aligned}
$$

provided that the series on the left-hand side is absolutely convergent and $a, b$, $x$ and $\vartheta$ are real numbers, and $i=\sqrt{-1}$. 
Proof. We begin by considering

$$
S:=e^{(a+i b) x} .
$$

Now let us express $a+i b$ in the polar coordinate and then Euler's formula:

$$
a+i b=r(\cos \vartheta+i \sin \vartheta)=r e^{i \vartheta},
$$

where

$$
r=\sqrt{a^{2}+b^{2}} \quad \text { and } \quad \vartheta=\arctan \left(\frac{b}{a}\right) .
$$

By using the Maclaurin expansion, we find from (2.2) and (2.3) that

$$
\begin{aligned}
S & =e^{(a+i b) x}=e^{\left(r e^{i \vartheta}\right) x}=\sum_{n=0}^{\infty} \frac{r^{n} x^{n}}{n !} e^{i \vartheta n} \\
& =\sum_{n=0}^{\infty} \frac{\left(a^{2}+b^{2}\right)^{\frac{n}{2}}}{n !} \cos (n \vartheta) x^{n}+i \sum_{n=0}^{\infty} \frac{\left(a^{2}+b^{2}\right)^{\frac{n}{2}}}{n !} \sin (n \vartheta) x^{n} .
\end{aligned}
$$

On the other hand, we have

$$
\begin{aligned}
S & =e^{(a+i b) x}=e^{a x} e^{i b x}=\left(\sum_{m=0}^{\infty} \frac{a^{m}}{m !} x^{m}\right)\left(\sum_{n=0}^{\infty} \frac{(i b)^{n}}{n !} x^{n}\right) \\
& =\sum_{m=0}^{\infty} \sum_{n=0}^{\infty} \frac{a^{m}(i b)^{n}}{m ! n !} x^{m+n}:=S_{e}+S_{o},
\end{aligned}
$$

where, upon separating the second sum into the even and odd parts, for convenience,

$$
S_{e}:=\sum_{m=0}^{\infty} \sum_{n=0}^{\infty} \frac{a^{m}(i b)^{2 n}}{m !(2 n) !} x^{m+2 n} \quad \text { and } \quad S_{o}:=\sum_{m=0}^{\infty} \sum_{n=0}^{\infty} \frac{a^{m}(i b)^{2 n+1}}{m !(2 n+1) !} x^{m+2 n+1} .
$$

Using the following easily-derivable formula (see, for example, [7, p. 6, Equation (28)]):

$$
(\lambda)_{2 n}=2^{2 n}\left(\frac{1}{2} \lambda\right)_{n}\left(\frac{1}{2} \lambda+\frac{1}{2}\right)_{n} \quad\left(n \in \mathbb{N}_{0}\right)
$$

and the following well known formal manipulation of double series (see, for example, [6, p. 57, Equation (7)]; for other manipulations, see also [2]):

$$
\sum_{m=0}^{\infty} \sum_{n=0}^{\infty} A(n, m)=\sum_{m=0}^{\infty} \sum_{n=0}^{\left[\frac{m}{2}\right]} A(n, m-2 n)
$$


we have

$$
\begin{aligned}
S_{e} & =\sum_{m=0}^{\infty} \sum_{n=0}^{\infty} \frac{(-1)^{n} a^{m} b^{2 n}}{m !(2 n) !} x^{m+2 n}=\sum_{m=0}^{\infty} \sum_{n=0}^{\infty} \frac{(-1)^{n} a^{m} b^{2 n}}{m ! 2^{2 n} n !\left(\frac{1}{2}\right)_{n}} x^{m+2 n} \\
& =\sum_{m=0}^{\infty} \sum_{n=0}^{\left[\frac{m}{2}\right]} \frac{(-1)^{n} a^{m-2 n} b^{2 n}}{(m-2 n) ! 2^{2 n} n !\left(\frac{1}{2}\right)_{n}} x^{m} .
\end{aligned}
$$

Using the following formula:

$$
(n-k) !=\frac{(-1)^{k} n !}{(-n)_{k}} \quad\left(0 \leqq k \leqq n ; n, k \in \mathbb{N}_{0}\right)
$$

and (2.6), after a little simplification, we get

$$
\begin{aligned}
S_{e} & =\sum_{m=0}^{\infty} \sum_{n=0}^{\left[\frac{m}{2}\right]} \frac{(-1)^{n} a^{m-2 n} b^{2 n}(-m)_{2 n}}{m ! 2^{2 n} n !\left(\frac{1}{2}\right)_{n}} x^{m} \\
& =\sum_{m=0}^{\infty} \frac{a^{m} x^{m}}{m !} \sum_{n=0}^{\left[\frac{m}{2}\right]} \frac{\left(-\frac{1}{2} m\right)_{n}\left(-\frac{1}{2} m+\frac{1}{2}\right)_{n}}{\left(\frac{1}{2}\right)_{n} n !}(-1)^{n}\left(\frac{b}{a}\right)^{2 n} .
\end{aligned}
$$

Using (1.2) for the inner sum, we finally obtain

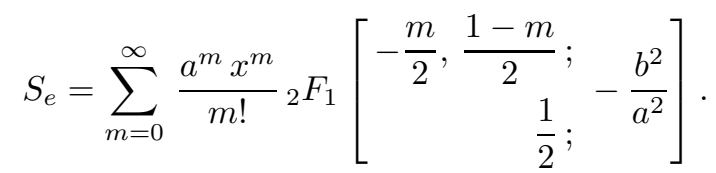

Similarly as in getting (2.10), we also have

$$
S_{o}=i x b \sum_{m=0}^{\infty} \frac{a^{m} x^{m}}{m !}{ }_{2} F_{1}\left[\begin{array}{c}
-\frac{m}{2}, \frac{1-m}{2} ; \\
\frac{3}{2} ;-\frac{b^{2}}{a^{2}}
\end{array}\right] .
$$

Hence, upon replacing $S_{e}$ and $S_{o}$ in (2.5) by (2.10) and (2.11), we immediately arrive at the desired formula (2.1). This completes the proof of Theorem 2 .

Remark 1. Writing the Euler formula $e^{i n \vartheta}=\cos (n \vartheta)+i \sin (n \vartheta)$ in $(2.1)$ and equating the real and imaginary parts, and then comparing the coefficients of $x^{n}$ in each of two resulting identities, we easily see the formulas in Theorem 1.

\section{Applications}

Here we consider some interesting applications of the results (1.11) and (1.12). The results to be established are asserted by the following theorem. 
Theorem 3. Each of the following formulas holds true.

$$
\begin{aligned}
& e_{0}^{x} F_{1}\left[\begin{array}{l}
-; \\
\frac{1}{2} ;
\end{array}-\frac{b^{2}}{4 a^{2}} x^{2}\right]=\sum_{m=0}^{\infty} \frac{x^{m}}{m !} \frac{\left(a^{2}+b^{2}\right)^{\frac{m}{2}}}{a^{m}} \cos (m \vartheta) ; \\
& e_{0}^{x} F_{1}\left[\frac{3}{2} ;-\frac{b^{2}}{4 a^{2}} x^{2}\right]=\sum_{m=1}^{\infty} \frac{x^{m-1}}{m !} \frac{\left(a^{2}+b^{2}\right)^{\frac{m}{2}}}{a^{m-1} b} \sin (m \vartheta) \text {; } \\
& (1-x)^{-2 \alpha}{ }_{2} F_{1}\left[\begin{array}{r}
\alpha, \alpha+\frac{1}{2} ; \\
\frac{1}{2} ;
\end{array}-\frac{b^{2} x^{2}}{a^{2}(1-x)^{2}}\right] \\
& =\sum_{m=0}^{\infty} \frac{(2 \alpha)_{m} x^{m}}{m !} \frac{\left(a^{2}+b^{2}\right)^{\frac{m}{2}}}{a^{m}} \cos (m \vartheta) \text {; } \\
& (1-x)_{2}^{-2 \alpha} F_{1}\left[\begin{array}{r}
\alpha, \alpha+\frac{1}{2} ; \\
\frac{3}{2} ;
\end{array} ; \frac{b^{2} x^{2}}{a^{2}(1-x)^{2}}\right] \\
& =\sum_{m=0}^{\infty} \frac{(2 \alpha)_{m} x^{m}}{m !} \frac{\left(a^{2}+b^{2}\right)^{\frac{m+1}{2}}}{a^{m} b}[\sin (m+1) \vartheta],
\end{aligned}
$$

where $\vartheta$ is the same as given in (1.13).

Proof. Denoting, for simplicity, the left-hand side of (3.1) by $L$ and expressing the two involved functions as series, after a little simplification, we have

$$
L=\sum_{m=0}^{\infty} \sum_{n=0}^{\infty} \frac{\left(-\frac{b^{2}}{a^{2}}\right)^{n} x^{m+2 n}}{\left(\frac{1}{2}\right)_{n} m ! n ! 2^{2 n}} .
$$

Using (2.7), we obtain

$$
L=\sum_{m=0}^{\infty} \sum_{n=0}^{\left[\frac{m}{2}\right]} \frac{\left(-\frac{b^{2}}{a^{2}}\right)^{n} x^{m}}{\left(\frac{1}{2}\right)_{n} n ! 2^{2 n}(m-2 n) !} .
$$

Using the following easily-derivable identities (see Equations (2.6) and (2.9)):

$$
(m-2 n) !=\frac{m !}{(-m)_{2 n}} \quad \text { and } \quad(-m)_{2 n}=2^{2 n}\left(-\frac{m}{2}\right)_{n}\left(-\frac{m}{2}+\frac{1}{2}\right)_{n},
$$

after a little simplification, we get

$$
L=\sum_{m=0}^{\infty} \frac{x^{m}}{m !} \sum_{n=0}^{\left[\frac{m}{2}\right]} \frac{\left(-\frac{m}{2}\right)_{n}\left(-\frac{m}{2}+\frac{1}{2}\right)_{n}}{\left(\frac{1}{2}\right)_{n} n !}\left(-\frac{b^{2}}{a^{2}}\right)^{n} .
$$


Expressing the inner series by using the functions ${ }_{p} F_{q}$ as in (1.2), we have

$$
L=\sum_{m=0}^{\infty} \frac{x^{m}}{m !}{ }_{2} F_{1}\left[\begin{array}{r}
-\frac{m}{2}, \frac{1-m}{2} ; \\
\frac{1}{2} ;
\end{array} ; \frac{b^{2}}{a^{2}}\right]
$$

Finally, using the known summation formula (1.11), we arrive at the righthand side of (3.1). This completes the proof of (3.1). The same argument as in proving (3.1) will easily establish the other three formulas (3.2) to (3.4).

If we take $b=a$ in Equations (3.1) to (3.4), we get the following known (see [1]) results asserted by the following theorem.

Theorem 4. Each of the following identities holds true.

$$
\begin{gathered}
e_{0}^{x} F_{1}\left[\begin{array}{l}
-; \\
\frac{1}{2} ;
\end{array}-\frac{x^{2}}{4}\right]=\sum_{m=0}^{\infty} \frac{x^{m}}{m !} 2^{\frac{m}{2}} \cos \left(\frac{m \pi}{4}\right) ; \\
e^{x} F_{1}\left[\frac{-;}{2} ;-\frac{x^{2}}{4}\right]=\sum_{m=1}^{\infty} \frac{x^{m-1}}{m !} 2^{\frac{m}{2}} \sin \left(\frac{m \pi}{4}\right) ;
\end{gathered}
$$

$$
(1-x)^{-2 \alpha} F_{1}\left[\begin{array}{r}
\alpha, \alpha+\frac{1}{2} ; \\
\frac{1}{2} ;
\end{array}-\frac{x^{2}}{(1-x)^{2}}\right]=\sum_{m=0}^{\infty} \frac{(2 \alpha)_{m} x^{m}}{m !} 2^{\frac{m}{2}} \cos \left(\frac{m \pi}{4}\right)
$$

$$
(1-x)^{-2 \alpha}{ }_{2} F_{1}\left[\begin{array}{r}
\alpha, \alpha+\frac{3}{2} ; \\
\frac{1}{2} ;
\end{array} ; \frac{x^{2}}{(1-x)^{2}}\right]=\sum_{m=0}^{\infty} \frac{(2 \alpha)_{m} x^{m}}{(m+1) !} 2^{\frac{m+1}{2}} \sin \left[\frac{(m+1) \pi}{4}\right] \text {. }
$$

Remark 2. Here it is interesting to point out that, in Equations (3.3) and (3.4), if we replace $x$ by $\frac{x}{\alpha}$ and let $\alpha \rightarrow \infty$, after a little simplification, we get the results (3.1) and (3.2), respectively.

Acknowledgements. This paper was supported by Basic Science Research Program through the National Research Foundation of Korea funded by the Ministry of Education, Science and Technology (2010-0011005). The secondnamed author would like to thank Prof. Dr. Jancy James, Vice Chancellor, Central University of Kerala, for granting him an academic leave to participate in Prof. Choi's research project funded by the above-mentioned institute. 


\section{References}

[1] W. N. Bailey, Products of generalized hypergeometric series, Proc. London Math. Soc. 28 (1928), no. 2, 242-254.

[2] J. Choi, Notes on formal manipulations of double series, Commun. Korean Math. Soc. 18 (2003), no. 4, 781-789.

[3] A. Erdélyi, W. Magnus, F. Oberhettinger, and F. G. Tricomi, Higher Transcendental Functions, Vol. 2, McGraw-Hill Book Company, New York, Toronto and London, 1953.

[4] A. P. Prudnikov, Yu. A. Brychkov, and O. I. Marichev, Integrals and Series, Vol. 3; More Special Functions, "Nauka", Moscow, 1986 (in Russian); Translated from the Russian by G. G. Gould, Gordon and Breach Science Publishers, New York, Philadelphia, London, Paris, Montreux and Melbourne, 1990.

[5] M. I. Qureshi, K. Quraishi, and H. M. Srivastava, Some hypergeometric summation formulas and series identities associated with exponential and trigonometric functions, Integral Transforms Spec. Funct. 19 (2008), no. 3-4, 267-276.

[6] E. D. Rainville, Special Functions, Macmillan Company, New York, 1960; Reprinted by Chelsea Publishing Company, Bronx, New York, 1971.

[7] H. M. Srivastava and J. Choi, Zeta and q-Zeta Functions and Associated Series and Integrals, Elsevier Science Publishers, Amsterdam, London and New York, 2012.

[8] H. M. Srivastava and H. L. Manocha, A Treatise on Generating Functions, Halsted Press (Ellis Horwood Limited, Chichester), John Wiley and Sons, New York, Chichester, Brisbane and Toronto, 1984.

Junesang Choi

Department of Mathematics

DongGuK University

GyeonguU 780-714, Korea

E-mail address: junesang@mail.dongguk.ac.kr

Arjun K. Rathie

Department of Mathematics

School of Mathematical \& Physical Sciences

Central University of Kerala

Riverside Transit Campus

Padennakad P.O. Nileshwar

KASARAGOD-671 328, INDIA

E-mail address: akrathie@gmail.com 